\title{
Esophageal Cancer Metastasis to the Eye: A Case Report and Literature Review
}

\author{
Ramzi A. Addas, Ahmed M. Alsabban, Abdullah A. Fallatah, Aqeel S. Binaqeel, \\ Salman W. Bafageeh
}

Department of Thoracic and General Surgery, King Abdulaziz Medical City, Jeddah, KSA

Email: ramziaddas79@gmail.com

How to cite this paper: Addas, R.A., Alsabban, A.M., Fallatah, A.A., Binaqeel, A.S. and Bafageeh, S.W. (2021) Esophageal Cancer Metastasis to the Eye: A Case Report and Literature Review. Open Journal of Thoracic Surgery, 11, 69-75.

https://doi.org/10.4236/ojts.2021.113009

Received: August 10, 2021

Accepted: September 19, 2021

Published: September 22, 2021

Copyright ( 2021 by author(s) and Scientific Research Publishing Inc. This work is licensed under the Creative Commons Attribution International License (CC BY 4.0). http://creativecommons.org/licenses/by/4.0/

\begin{abstract}
Ocular metastasis is an uncommon occurrence that usually has a poor prognosis. The most frequent causes of intraocular metastases as reported in the literature are breast and lung cancers. Intraocular metastasis originating from esophageal cancer is very rare. We discuss a case of 58 years old man known case of adenocarcinoma of the esophagogastric junction presenting with ocular metastasis in King Abdulaziz Medical City, Jeddah, Saudi Arabia. Clinical symptoms led to the investigations and diagnosis of the ocular metastasis which without these symptoms it would have been missed. We concluded that a careful and good review of the whole body is essential in the optimal diagnosis of metastatic lesions especially when it comes to a rare metastasis like ocular metastasis from esophageal cancer.
\end{abstract}

\section{Keywords}

Esophageal Cancer, Ocular Metastasis, Orbital MRI, Palliative Therapy

\section{Introduction}

The most common pattern of esophageal cancer metastases is to the lymph nodes, lung, liver, bones, adrenal glands, and brain. On the other hand, unexpected metastasis spread to uncommon sites has increasingly been reported and consequently affected the pathway of its diagnosis, staging as well as management.

Esophageal cancer is one of the most fatal cancers globally ranking 8th among other cancers due to its extremely aggressive nature and poor life expectancy. In terms of cancer-related death, it is the sixth most deadly of all cancers [1]. Unexpected metastasis to unusual sites such as skin, eye, muscle, and heart has been reported, affecting the diagnosis, staging, and treatment. Ocular metastasis is a 
rare condition and is associated with a poor prognosis. Herein, we present a case of a patient with esophageal cancer with ocular metastasis in our institute (King Abdulaziz Medical City, Jeddah, Saudi Arabia) hoping for a better understanding of this rare presentation of the esophageal cancers, its nature, diagnosis and management.

\section{Case Presentation}

A 58-year-old man known case of diabetes mellitus and hypertension, heavy smoker was initially admitted to king Abdulaziz medical city, National Guard Hospital, Jeddah, Saudi Arabia complaining of difficulty swallowing. He had a history of three months of dysphagia to solid food. The patient had an upper GI endoscopy which showed a mass at the gastroesophageal junction. Biopsy was taken from the lesion which showed a moderately differentiated adenocarcinoma. For the rest of the esophagus, stomach, and duodenum, they appeared normal.

As there was no obvious metastasis at the staging at this level the treatment plan was to start neoadjuvant chemotherapy followed by esophagectomy. The patient was started on FLOT (Docetaxel, oxaliplatin, leucovorin, and 5-fluorouracil) and after finishing his neoadjuvant treatment he was admitted for pre-operative testing. PET/CT was performed and unfortunately, it demonstrated adrenal gland metastasis as well as a new deposit located at the seventh thoracic vertebral body despite improvement in the tumor size and avidity.

During this period the patient was complaining of left eye pain, headache, vomiting, decreased vision, and photophobia. Upon left eye examination, the visual acuity was 6/9. He had congested eye, severe cornea edema, hemorrhagic hypopyon, keratic precipitates, hazy fundus view, sluggish papillary reaction. He had swollen left eyelid with conjunctival chemosis and corneal haziness, the pupil was not reactive to light [Figure 1].

The patient was on full anti-glaucoma and Diamox and still felt severe left eye pain and can't sleep. He was seen in the ophthalmology department clinic and diagnosed with left neovascular inflammatory glaucoma and uveitis probably due to iris metastasis. An orbital MRI was performed which showed left orbital metastasis. The orbits show left extraconal orbital enhanced mass which is seen inseparable from the lateral rectus muscle associated with abnormal thickened enhancement of the left orbital nerve, there is subtle proptosis noted at the left orbit as well [Figure 2] and he was diagnosed in the ophthalmology clinic with ocular metastasis originating from his esophageal cancer.

With all of that progression and metastasis, the decision was made to put the patient on palliative therapy as there was no role for surgical treatment with the presence of these multiple site's metastatic lesions. Unfortunately, the disease continued to progress with new metastasis to other body organs including the brain and the vertebrae, the patient was on palliative chemotherapy and radiotherapy including craniospinal radiation. The general condition deteriorated further, and the patient passed away after around three months from the diag- 
nosis of his metastasis. Although it is not common, this case shows that rare metastasis occurs (like eye metastasis from esophageal cancer) and a careful and good review of the whole body is essential in addition to a high index of suspicion as well as thinking outside the box.
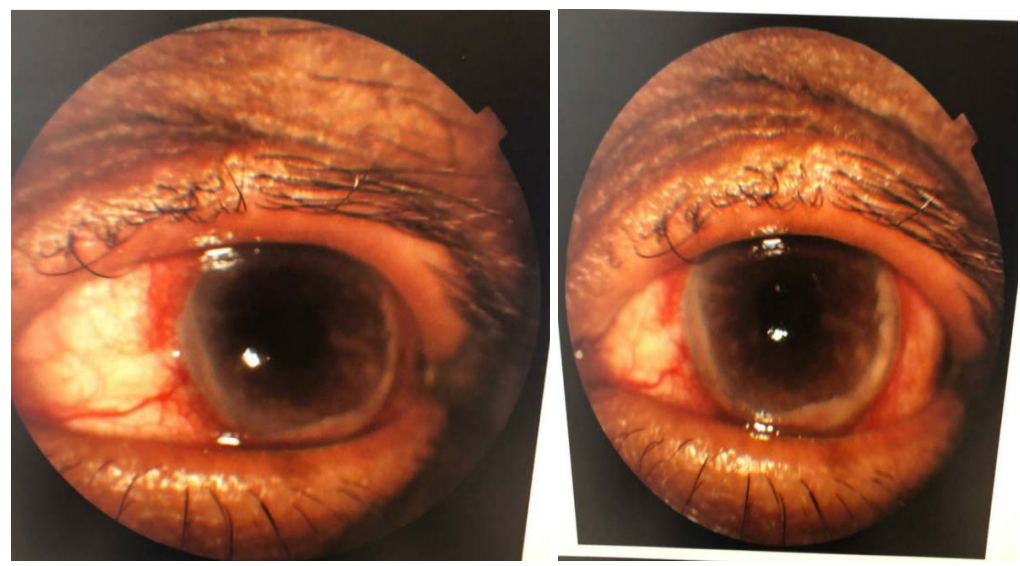

Figure 1. Eye examination of our patient showed swollen left eyelid with conjunctival chemosis and corneal haziness, severe cornea edema, with hemorrhagic hypopyon and a pupil which is not reactive to light.

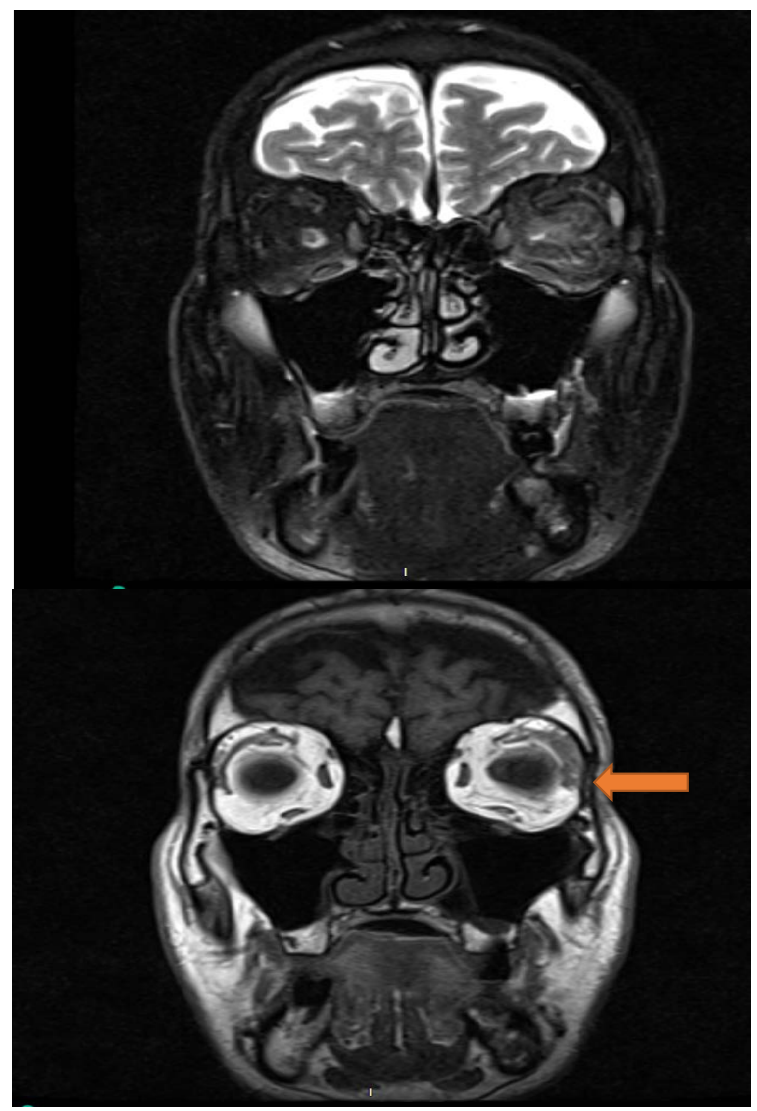

Figure 2. MRI orbits show left extraconal orbital enhanced mass (arrow) which is seen inseparable from the lateral rectus muscle associated with abnormal thickened enhancement of the left orbital nerve, there is subtle proptosis noted at the left orbit as well. 


\section{Discussion}

Historically, distal metastases of cancers have been considered the most potent challenge in achieving a real advance in cancer management. In addition, being responsible for $90 \%$ of cancer-related deaths [2] [3]. The impact of esophageal cancer regional and distal metastases on treatment and survival has been widely studied. On the other hand, unexpected metastases have been only investigated through sporadic case reports and small case series studies like the ocular metastasis which we are representing in this paper.

Esophageal cancer distant metastases can leave the esophagus via three possibilities: lymphatic, venal, or arterial routes. The complex anatomical pathway of the esophagus lymphatic system including the lymphatic nodal skip (retrograde and bidirectional) spread [4] [5] can also explain the possibility of the random distribution of the metastases in these cases. The absence of serosal coating and the presence of periesophageal adventitia that connects it with the mediastinal structures have an important impact on the lymph node metastasis frequency and tumor ingrowth into the surrounding and distal organs. The venous routes will pass to the pulmonary system via the vena cava explaining the pulmonary metastases, or in the portal system explaining the hepatic metastases. Isolated distal metastases in terminal organs (such as the skin, penis, lips, or retina, etc.) can be hardly explained based on lymphatic or venal routs alone. The expected route of those unexpected metastases may be explained by the arterial pathway [6]. Regarding the esophagus, there are multiple arterial sources (shared vasculature) starting from the inferior thyroid artery to the splenic artery across different arterial supplies between them. The arterial route could be how this tumor embolism liberates and pass toward the main artery to follow an anatomical direction and finally reach the distal terminal organs [6].

Ocular metastasis is a rare entity. In one of the reported series of orbital tumors, metastases accounted for $7 \%$ of the total orbital tumors in adults [7]. Recently, the incidence of ocular metastasis has increased [8]. Several factors may account for this increase including the general improvement in the treatment that led to an increase in the median survival of cancer patients but in turn that has increased the chances for development of metastatic lesions in unusual sites such as the orbit. Another factor is the advances in the diagnosis and application of serological and molecular diagnostic imaging techniques which led to the increased detection of such lesions. Finally, the increased volume of medical literature on orbital metastasis has increased the awareness of these lesions. The actual incidence of orbital metastasis is believed to be higher than that described in the literature. Underestimation of the true incidence can be attributed to several factors such as small orbital lesions may remain asymptomatic, and general debility in patients with the widespread disease may mask the orbital symptoms leading to a low rate of referral to ophthalmologists [9] [10].

The majority of metastatic cancers to the eye arise from the lung, breasts (48\%) and prostate (12\%) [7]. Regarding metastases originating from the GI 
tract, it accounts for only $6 \%-7 \%$ of all reported cases [11].

Orbital swelling and proptosis are the most common manifestations of primary eye malignancy. These could be associated with a protrusion, loss of visual acuity and field, pain and watering which can present acutely or in a progressive way [12]. In some reported cases, orbital metastases presented as orbital cellulitis or abscess [7].

Treatment of ocular metastases includes radiotherapy, chemotherapy, laser surgery, anti-vascular endothelial growth factor administration and eye enucleation which should be considered as a last resort [13] [14].

Regarding the diagnosis of ocular metastasis, first which is very important is a clear and thorough history that could lead to the suspicion of the ocular metastasis. Then, ophthalmic examination with slit-lamp bio-microscopy and indirect ophthalmoscopy are important diagnostic tools for use upon initial examination. Orbital imaging using CT and MRI are the principal means of diagnosing and evaluating orbital metastasis. The definitive diagnosis requires tissue samples. Fine needle aspiration has been advocated as a good diagnostic modality. However, there have been reports of dissemination of tumor cells with the procedure, and there is a risk of global injury, although the likelihood of these complications is low [9].

The prognosis of patients with a metastatic ocular lesion is generally poor.

Char DH et al. reported in their study that the mean and the 2-year survival rates were $1.3 \%$ and $27 \%$, respectively [15]. Other studies showed that Survival after diagnosis of orbital metastases ranges from 13 months to 1.5 years, with variation due to histologic type [16] [17].

\section{Conclusions}

This unusual case brings up important points to consider. Even though some metastases are rare, they can present anywhere in the body. Therefore, a careful and good review of the whole body is essential in the optimal diagnosis of metastatic lesions. The ocular lesions in patients with a history of cancer should give special consideration in clinical practice. Moreover, to avoid misdiagnosis, thorough systemic and specialist examinations are needed.

Regarding this type of patient and since more than half of patients have another metastasis when the ocular lesion is discovered, treatment is usually not curative.

\section{Conflicts of Interest}

The authors declare no conflicts of interest regarding the publication of this paper.

\section{References}

[1] Zhang, Y. (2013) Epidemiology of Esophageal Cancer. World Journal of Gastroenterology, 19, 5598-606. https://doi.org/10.3748/wjg.v19.i34.5598 
[2] Chaffer, C.L. and Weinberg, R.A. (2011) A Perspective on Cancer Cell Metastasis. Science, 331, 1559-1564. https://doi.org/10.1126/science.1203543

[3] Gupta, G.P. and Massague, J. (2006) Cancer Metastasis: Building a Framework. Cell, 127, 679-695. https://doi.org/10.1016/j.cell.2006.11.001

[4] Weijs, T.J., Goense, L., van Rossum, P.S., Meijer, G.J., van Lier, A.L.H.M.W., Wessels, F.J., et al. (2017) The Peri-Esophageal Connective Tissue Layers and Related Compartments: Visualization by Histology and Magnetic Resonance Imaging. Journal of Anatomy, 230, 262-271. https://doi.org/10.1111/joa.12552

[5] Maingot, R., Zinner, M. and Ashley, S.W. (2007) Maingot's Abdominal Operations. 11th Edition, McGraw-Hill, London, New York.

[6] Shaheen, O., Ghibour, A. and Alsaid, B. (2017) Esophageal Cancer Metastases to Unexpected Sites: A Systematic Review. Gastroenterology Research and Practice, 2017, Article ID: 1657310. https://doi.org/10.1155/2017/1657310

[7] George, N.M., Charles, M.P., Wade, A.R., Patel, K.G., Lentsch, E.J. and Eiseman, A.S. (2015) Esophageal Adenocarcinoma and Urothelial Carcinoma Orbital Metastases Masquerading as Infection. Orbit, 34, 51-55. https://doi.org/10.3109/01676830.2014.963879

[8] Dieing, A., Schulz, C.O., Schmid, P., Roever, A.C., Lehenbauer-Dehm, S., Jehn, C., et al. (2004) Orbital Metastases in Breast Cancer: Report of Two Cases and Review of the Literature. Journal of Cancer Research and Clinical Oncology, 130, 745-748. https://doi.org/10.1007/s00432-004-0606-3

[9] Goldberg, R.A., Rootman, J. and Cline, R.A. (1990) Tumors Metastatic to the Orbit: A Changing Picture. Survey of Ophthalmology, 35, 1-24. https://doi.org/10.1016/0039-6257(90)90045-W

[10] Eldesouky, M.A. and Elbakary, M.A. (2015) Clinical and Imaging Characteristics of Orbital Metastatic Lesions among Egyptian Patients. Clinical Ophthalmology, 9, 1683-1687. https://doi.org/10.2147/OPTH.S87788

[11] Lee, J.Y., Lee, H.J., Jung, M.S. and Kim, S.Y. (2010) Metastatic Esophageal Squamous Cell Carcinoma to the Orbit and Periorbit Masquerading as Periorbital Abscess. Korean Journal of Ophthalmology, 24, 123-125. https://doi.org/10.3341/kjo.2010.24.2.123

[12] Moss, H.M. (1962) Expanding Lesions of the Orbit. A Clinical Study of 230 Consecutive Cases. American Journal of Ophthalmology, 54, 761-770.

https://doi.org/10.1016/0002-9394(62)94157-0

[13] Giuliari, G.P. and Sadaka, A. (2012) Uveal Metastatic Disease: Current and New Treatment Options. Oncology Reports, 27, 603-607. https://doi.org/10.3892/or.2011.1563

[14] Valenzuela, A.A., Archibald, C.W., Fleming, B., Ong, L., O’Donnell, B., John Crompton, J., et al. (2009) Orbital Metastasis: Clinical Features, Management and Outcome. Orbit, 28, 153-159. https://doi.org/10.1080/01676830902897470

[15] Char, D.H., Miller, T. and Kroll, S. (1997) Orbital Metastases: Diagnosis and Course. British Journal of Ophthalmology, 81, 386-390.

https://doi.org/10.1136/bjo.81.5.386

[16] Shields, C.L., Shields, J.A. and Peggs, M. (1988) Tumors Metastatic to the Orbit. Ophthalmic Plastic \& Reconstructive Surgery, 4, 73-80. https://doi.org/10.1136/bjo.81.5.386

[17] Magliozzi, P., Strianese, D., Bonavolontà, P., Ferrara, M., Ruggiero, P., Carandente, R., et al. (2015) Orbital Metastases in Italy. International Journal of Ophthalmology, 8, 1018-1023. 


\section{Abbreviations}

\section{GI: gastrointestinal;}

FLOT: fluorouracil, leucovorin, oxaliplatin, docetaxel;

MRI: magnetic resonance imaging;

CT: computed tomography;

PET/CT: positron emission tomography-computed tomography. 